\title{
Eficacia del uso de un enjuague con aceites esenciales para el control del biofilm dental.
}

\section{Efficacy of the use of a rinse with essential oils for the control of dental biofilm.}

\author{
Dolores De la Cruz Cardoso, ${ }^{*, \ddagger}$ Evelyn Nineth Pérez Madrigal, \\ Angélica Cassandra Pérez Sotelo, ${ }^{\S}$ Armando Cervantes Sandoval*
}

\section{RESUMEN}

Introducción: Se ha documentado que, para tener mejores resultados en el control del biofilm oral, es necesario el uso de controles químicos, antes o después del cepillado dental. Entre éstos, encontramos los enjuagues de aceites esenciales. Objetivo: Determinar la actividad del enjuague con aceites esenciales antes o después del cepillado, en el control del biofilm dental. Material y métodos: El estudio se realizó con 27 voluntarios. Los participantes fueron seleccionados y agrupados. El grupo 1 fue el control, que empleó su técnica de cepillado habitual y dos grupos experimentales que, además del cepillado con dentífrico, usaron un enjuague de aceites esenciales durante ocho días, ya sea antes (grupo 2) o después del cepillado dental (grupo 3). La cuantificación del número de superficies teñidas en los tres grupos, tanto el estado inicial como posterior al uso de los enjuagues, se hizo con el índice de O’Leary y un revelador de placa tritonal, el cual permitió la observación del biofilm de forma clínica, así como su grado de patogenicidad. Resultados y conclusión: El análisis estadístico estableció que no existe diferencia significativa entre no usar y usar el enjuague con aceites esenciales antes o después del cepillado.

Palabras clave: Aceites esenciales, control biofilm, índice de higiene, cepillado.

\section{ABSTRACT}

Introduction: It has been documented that, to have better results in the control of oral biofilm, it is necessary to use chemical control, before or after tooth brushing. Among these, we find the essential oil rinses. Objective: To determine the activity of the rinse with essential oils before or after brushing, in the control of the dental biofilm. Material and methods: The study was conducted with 27 volunteers. The participants were selected and grouped. Group 1 was the control, which used its usual brushing technique and two experimental groups that, in addition to brushing with toothpaste, used a mouthwash of essential oils, for eight days, either before (group 2) or after tooth brushing (group number 3). For the quantification of the number of stained surfaces in the three groups, to record both the initial and subsequent state of the use of the rinses, it was done with the O'Leary index and a tritonal developer, which allowed the observation of the biofilm of clinical form, as well as its degree of pathogenicity. Results and conclusion: The statistical analysis established that there is no significant difference between not using and using the rinse with essential oils before or after brushing.

Keywords: Essential oils, biofilm control, hygiene index, brushing.

\section{INTRODUCCIÓN}

Se e ha documentado que, para tener mejores resultados en el control del biofilm oral, es necesaria la implementación del uso de algún medio de control químico. ${ }^{1,2}$ Entre éstos se encuentran los agentes antiadhesivos, que retrasan la proliferación bacteriana y los antimicrobianos que alteran la estructura patogénica del biofilm. El dentífrico fluorurado y los enjuagues bucales son los dos principales medios de control químico. ${ }^{3}$ Existen diferentes tipos de colutorios, entre los más utilizados se encuentran los elaborados con aceites esenciales (timol, mentol, eu-

\footnotetext{
* Universidad Nacional Autónoma de México, Facultad de Estudios Superiores Zaragoza, Ciudad de México, México.

‡ Unidad de Investigación en Cardiología UNAM-INC (Instituto Nacional de Cardiología).

$\S$ Cirujano Dentista, UNAM.
}

Recibido: 19 Febrero 2020. Aceptado para publicación: 07 Septiembre 2020.

Citar como: De la Cruz CD, Pérez MEN, Pérez SAC, Cervantes SA. Eficacia del uso de un enjuague con aceites esenciales para el control del biofilm dental Revista ADM. 2020; 77 (5): 247-451. https://dx.doi.org/10.35366/96143 
caliptol). ${ }^{4}$ Cuyo uso, posterior al cepillado, ha mostrado buena efectividad en el control del biofilm dental. ${ }^{5-8}$

Así mismo, hay estudios que muestran que el uso previo al cepillado de triclosán, copolímeros y fluoruro puede dar buenos resultados en el control del biofilm. ${ }^{9-14}$

De esta manera, el objetivo de nuestra investigación es comparar la reducción del número de superficies con biofilm, que se presenta con el uso de los aceites esenciales previo al cepillado dental, respecto de la que encontramos al usarlos posterior al mismo.

\section{MATERIAL Y MÉTODOS}

Se hizo un estudio longitudinal, prospectivo, analítico y comparativo. La selección de la población participante se llevó a cabo a partir de un cuestionario para evaluar la presencia de algunas enfermedades que pudieran alterar la producción de saliva y para consignar el estado del cepillo, así como la frecuencia y tiempo del cepillado. Este último dato se tomó como base para el establecimiento de tres grupos, en los cuales se distribuyeron, de manera equitativa, tres individuos por cada tiempo de exposición al fluoruro por dentífrico, de acuerdo con lo que indicaron los participantes (180, 240 y 360 segundos). No se intervino ninguno de los hábitos higiénicos declarados por los participantes. De esta manera, los grupos experimentales introdujeron el uso de dos enjuagues con aceites esenciales, uno en la mañana y otro en la noche. El grupo 2, lo llevó a cabo antes del cepillado, mientras que el grupo 3 lo hizo después del

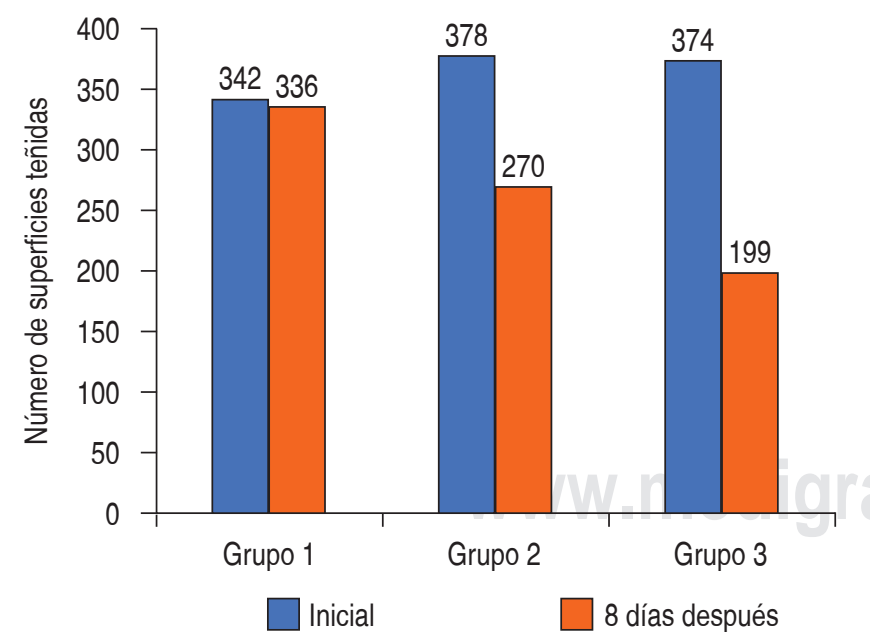

Figura 1: Número de superficies teñidas por grupo inicial y ocho días después del estudio. Población universitaria entre 18-23 años. UNAM FES Zaragoza.
Tabla 1: Número inicial y final de superficies teñidas

por color de tinción. Grupo 1. Población universitaria entre 18-23 años. UNAM FES Zaragoza.

\begin{tabular}{lrrc} 
Tiempo & Rosa & Azul & Morado \\
\hline Inicial & 293 & 26 & 23 \\
Ocho días después & 285 & 35 & 16 \\
Diferencia & 8 & -9 & 7 \\
\hline
\end{tabular}

mismo. El grupo 1 fue el control, por lo que sólo llevaron a cabo su higiene habitual.

Se aplicó el índice de O'Leary a cada uno de los participantes, tanto al inicio como al final del estudio. Las comparaciones del efecto de la aplicación de los aceites esenciales, y en su caso del grupo control, se llevaron a efecto tomando en consideración únicamente el conteo de las superficies que nos arrojó el índice. La tinción utilizada fue tritonal, la cual nos permitió observar el grado de patogenicidad en la que se encontraba el biofilm durante el estudio. Fueron incluidos alumnos de la carrera de cirujano dentista de la Facultad de Estudios Superiores Zaragoza, UNAM, que participaron de manera voluntaria después de recibir una plática acerca de las condiciones y objetivos del estudio y que entregaron el consentimiento informado firmado antes del inicio de la investigación. Alumnos que indicaron ser fumadores activos, que tuvieran algún tipo de aparatología fija u ortodoncia, o que presentaran alguna enfermedad sistémica que pudiera alterar la producción y composición de la saliva, fueron excluidos.

Se aplicaron técnicas de estadística descriptiva, así como técnicas no paramétricas (ANCOVA) y paramétricas (ANOVA).

\section{RESULTADOS}

Participó un total de 27 alumnos, con un rango de edad entre 18 y 23 años, pertenecientes a todos los años de la carrera.

Los resultados clínicos del estudio de cada grupo participante, tanto inicial como final, pueden observarse en la Figura 1. En el grupo 1, o control, registramos un número de superficies teñidas al final del estudio similar al inicial. Por otra parte, en el grupo 2, constituido por individuos que usaron aceites esenciales antes del cepillado, encontramos una diferencia de 108 superficies teñidas entre la medición inicial y la final. Mientras que el grupo 3, quienes usaron los aceites esenciales después del 
cepillado, se determinó una diferencia de 175 superficies, que fue prácticamente la mitad del promedio inicial de caras teñidas.

Así mismo, mediante el uso del revelador tritonal, se puede observar en la Tabla 1 un aumento en las superficies teñidas en color azul. El grupo 2 refleja, ocho días después del inicio del estudio, una disminución del número de superficies teñidas en color rosa y azul (Tabla 2). Después de utilizar el enjuague con aceites esenciales en el grupo 3 , se observa una reducción notable en las superficies teñidas en color rosa y morado (Tabla 3).

Análisis estadístico. Al realizar un análisis de covarianza (ANCOVA), observamos que los grupos que utilizaron el enjuague con aceites esenciales antes y después del cepillado dental, muestran similitud entre sí. No obstante, se puede observar una diferencia entre el grupo control y el grupo 3, el cual utilizó el enjuague con aceites esenciales después del cepillado (Figura 2). Asimismo, se llevó a efecto un análisis de comparaciones múltiples (ANOVA), lo que nos condujo a rechazar la hipótesis planteada en el presente estudio. El análisis mostró que no hay diferencia estadísticamente significativa $(p>0.05)$ entre usar el enjuague de aceites esenciales antes o después del cepillado dental, e incluso no usarlo (Tabla 4). Se realizó un análisis de varianza (ANOVA) con las diferentes variables que fueron consideradas posibles determinantes en el estudio, como: condiciones del cepillo dental, exposición de fluoruro/día y veces de cepillado/día, tras lo cual se concluyó que no hay diferencia estadísticamente significativa. Por lo que ninguna de las variables antes mencionadas influye en los resultados.

\section{DISCUSIÓN}

El análisis de covarianza (ANCOVA) de los resultados del uso del enjuague con aceites esenciales, posterior al cepillado dental (grupo 3), estableció una diferencia estadísticamente significativa respecto al grupo control.

Tabla 2: Número inicial y final de superficies teñidas por color de tinción. Grupo 2. Población universitaria entre 18-23 años. UNAM FES Zaragoza.

\begin{tabular}{lrcc} 
Tiempo & Rosa & Azul & Morado \\
\hline Inicial & 319 & 37 & 22 \\
Ocho días después & 238 & 20 & 12 \\
Diferencia & 81 & 17 & 10 \\
\hline
\end{tabular}

Tabla 3: Número inicial y final de superficies teñidas por color de tinción. Grupo 3. Población universitaria entre 18-23 años. UNAM FES Zaragoza.

\begin{tabular}{lccc} 
Tiempo & Rosa & Azul & Morado \\
\hline Inicial & 329 & 17 & 28 \\
Ocho días después & 166 & 21 & 12 \\
Diferencia & 163 & -4 & 16 \\
\hline
\end{tabular}

La hipótesis de esta investigación fue rechazada por un análisis estadístico más estricto, como lo es el análisis de varianza (ANOVA), ya que la reducción del número de las superficies con biofilm fue igual en los tres grupos $(p$ $>0.05)$.

Sin embargo, clínicamente se observó una mayor reducción al usarlo después del cepillado dental, datos que concuerdan con los estudios realizados por Cortelli, Lynch y Araujo. ${ }^{15-17}$ Lo cual sugiere que el uso del enjuague con aceites esenciales, después del cepillado, podría ser útil en el control del biofilm dental.

En nuestros resultados se observó una mayor reducción del número de superficies con biofilm dental de menor patogenicidad (rosa). Esto es consistente con lo encontrado por Quintas, quien observó que los aceites esenciales tienen un mayor efecto sobre las capas más superficiales, ya que las que se encuentran más profundas presentan una estructura que impide la penetración del antiséptico dentro de la compleja estructura de la red bacteriana, la que constituye el biofilm dental, ${ }^{4}$ lo que pudo haber ocurrido en la poca disminución del biofilm acidogénico (azul) y el de más de 48 horas (morado) que, como sabemos, cuenta con una mayor densidad en su estructura.

Por otro lado, sabemos que el cepillado dental constituye el método de control más eficaz sobre el biofilm supragingival, si se realiza de manera adecuada. ${ }^{18-20}$ Los resultados iniciales de nuestro estudio denotan una higiene deficiente en la población participante, de acuerdo con la clasificación del índice de O'Leary. Lo cual es indicativo de que esta población no realiza adecuadamente su cepillado, y de que el número de veces que lo hacen no influye en la reducción del número de superficies con biofilm dental. Incluso al visualizar los resultados finales, tenemos que el número de superficies teñidas de los grupos experimentales difiere sólo 67 unidades del resultado inicial, sin importar el número de veces de cepillado, y si el enjuague se usó antes o después de 
éste. Asimismo, si observamos el comportamiento del grupo control, podemos afirmar que lo que influyó en la modesta disminución del número de superficies con biofilm, en el caso de los grupos experimentales, fue el uso de aceites esenciales y no el número de veces de cepillado que los participantes realizaron.

Como lo señalamos al inicio, el tiempo de exposición a fluoruro fue estandarizado en todos los grupos, a partir del tiempo de cepillado que declararon, por lo que la exposición a fluoruro no fue una variable que influyera en la disminución del biofilm dental.

Tangade indica que para tener un buen control del biofilm dental, es necesario realizar la higiene oral con un cepillo dental en excelentes condiciones, pues señala que a medida que los cepillos se desgastan, su capacidad para el control del biofilm disminuye. ${ }^{21}$ En el presente estudio se encontró que las condiciones del cepillo no tuvieron relación con la eficacia de la higiene oral, pues a pesar de que los participantes mencionaron que su cepillo estaba en buenas condiciones, la higiene oral inicial fue deficiente. Por lo que consideramos que el esmero con el que se hace la higiene es determinante para alcanzar, cuando menos, una higiene aceptable.

Aunque el conocimiento que los alumnos tenían con respecto a la higiene y el control del biofilm dental no fue una variable considerada en el estudio, es importante señalar que eran estudiantes de diferentes años de la carrera de cirujano dentista, que ya cuentan con conte-

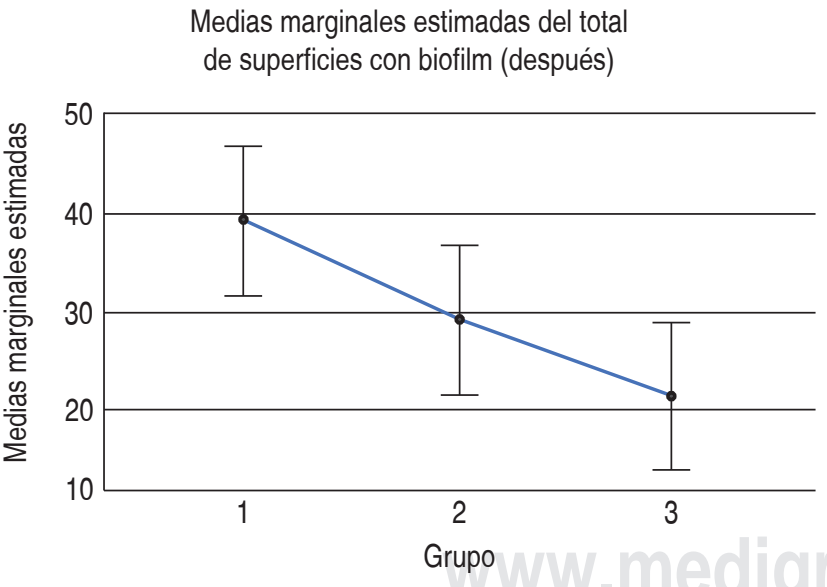

La covariables que aparecen en el modelo se evalúan en los valores siguientes: total de superficies con biofilm (antes) $=40.41$. Barras de error: IC95\%.

Figura 2: Comparación del estado de higiene oral inicial y ocho días después entre grupos. Población universitaria entre 18-23 años. UNAM FES Zaragoza.
Tabla 4: Diferencia de medias del número de superficies con biofilm por grupo. Prueba de Tukey. Población universitaria entre 18-23 años. UNAM FES Zaragoza.

\begin{tabular}{ccc} 
Grupo & $\mathrm{n}$ & Subconjunto \\
\hline 1 & 9 & 37.61 \\
2 & 9 & 36.00 \\
3 & 9 & 31.89 \\
Significancia & & 0.538 \\
\hline
\end{tabular}

nidos relacionados al control de biofilm oral, auxiliares de higiene y factores relacionados con la acumulación del biofilm dental. A pesar de ello, mostraban un descuido en su higiene oral. Consideramos que los resultados podrían haber sido más favorables que los registrados, sobre todo porque son contenidos académicos aplicados por los alumnos en la clínica.

\section{CONCLUSIÓN}

Podríamos afirmar que en esta población no se observó diferencia estadísticamente significativa entre usar o no los aceites esenciales antes o después del cepillado dental. Además, notamos que el número de veces de cepillado no influyó en la reducción del biofilm dental, y que las condiciones del cepillo no influyeron en la eficacia de la higiene oral de los participantes.

\section{BIBLIOGRAFÍA}

1. Mohamed J, Shankargouda P, Jagadish H, Shilpa H, Elna P, Sukumaran A. A chemical plaque control strategies in the prevention of biofilm associated oral diseases. J Contemp Dent Pract. 2016; 17 (4): 337-343.

2. Sanz SI. Control superior de los biofilms orales. Gaceta Dental. 2017; 292: 3-13.

3. Lindhe J, Karring T, Lang NP. Periodontología clínica e implantología odontológica. 5a. Buenos Aires: Ed. Médica Panamericana. 2009.

4. Quintas V, Prada I, Carreira MJ, Suárez D, Balsa C, Tomás I. In situ antibacterial activity of essential oils with and without alcohol on oral biofilm: a randomized clinical trial. Front Microbiol. 2017; 8: 1-16.

5. Kulkarni P, Singh DK, Jalaluddin M, Mandal A. Comparative evaluation of antiplaque efficacy between essencial oils with alcohol-based and chlorhexidine with nonalcohol-based mouthrinses. J Int Soc Prev Community Dent. 2017; 7 (1): 36-41.

6. Marchetti E, Teco S, Caterina E, Casalena F, Quinzi V, Mattei A et al. Alcohol-free essential oils containing mouthrinse efficacy on three-day supragingival plaque regrowth: a randomized crossover clinical trial. Trials. 2017; 18: 154.

7. Charugundla BR, Anjum S, Mocherla M. Comparative effect of fluoride, essential oil and chlorhexidine mouth rinses on dental 
plaque and gingivitis in patients with and without dental caries: a randomized controlled trial. Int J Dent. 2015; 12 (2): 104-109.

8. Alshehri FA. The use of mouthwash containing essential oils (LISTERINE $®$ ) to improve oral health: a systematic review. Saudi Dent J. 2018; 30 (1): 2-6.

9. Schiff T, Borden LC. The effect of a new experimental prebrushing dental rinse on plaque removal. J Clin Dent. 1994; 4 (4): 107-110.

10. Lobene RR, Singh SM, Petrone ME, Volpe AR, Chaknis P. Clinical efficacy of a triclosan/copolymer pre-brush rinse. J Clin Dent. 1992; 3 (2): 54-58.

11. Balanyk TE, Galustians HJ. Antiplaque efficacy of a prebrushing rinse. Am J Dent. 1992; 5 (1): 46-48.

12. Rustogi KN, Petrone DM, Singh SM, Volpe AR, Tavss E. Clinical study of a pre-brush rinse and a triclosan/copolymer mouthrinse: effect on plaque formation. Am J Dent. 1990: 67-69.

13. Deasy MJ, Gattista G, Rustogi KN, Volpe AR. Aintiplaque efficacy of a triclosan/copolymer prebrush rinse: a plaque prevention clinical study. Am J Dent. 1992; 5 (2): 91-94.

14. Hunter L, Addy M, Moran J, Kohut B, Hovliaras CA, Newcombe RG. A study of a pre-brushing mouthrinse as an adjunct to oral hygiene. J Periodontol 2000. 1994; 65 (8): 762-765.

15. Cortelli SC, Cortelli JR, Wu MM, Simmons K, Charles CA. Comparative antiplaque and antigingivitis efficacy of a multipurpose essential oil-containing mouthrinse and a cetylpyridinium chloridecontaining mouthrinse: a 6-month randomized clinical trial. Quintessence Int. 2012; 43: 82-94.
16. Lynch MC, Cortelli CS, McGuire AJ, Zhang J, Ricci-Nittel D, Mordas JC et al. The effects of essencial oils mouthwash with or without alcohol on plaque and gingivitis: a randomized controlled clinical study. BMC Oral Health. 2018; 18: 6.

17. Araujo MW, Charles CA, Weinstein RB, McGuire JA, Parikh-Das AM et al. Meta-analysis of the effect of an essencial oil-containing mouthrinse on gingivitis and plaque. J Am Dent. 2015; 146 (8): 610-622.

18. Maddi A, Frank A. Scannapieco. Oral biofilms, oral and periodontal infections, and systemic disease. Am J Dent. 2013; 26 (5): 249-254.

19. Cunha-Cruz J, Milgrom P, Shirtcliff RM, Huebner CE, Ludwig S, Allen $G$ et al. Everybody brush!": protocol for a parallel-group randomized controlled trial of a family-focused primary prevention program with distribution of oral hygiene products and education to increase frequency of toothbrushing. JMIR Res Protoc. 2015; 4 (2): e58.

20. Twetman S. Prevention of dental caries as a non-communicable disease. Eur J Oral. 2018; 126: 19-25.

21. Tangade PS, Farooq AS, Ravishankar TL, Tirth A, Pal S. Is plaque removal efficacy of toothbrush related to bristle flaring? A 3-month prospective parallel experimental study. Ethiop Health Sci. 2013; 23 (3): 255-264.

Correspondencia:

Dolores De la Cruz Cardoso

E-mail: dolorescc53@gmail.com 